\title{
Visualization of Haplotype Sharing Patterns in Pedigree Samples
}

\author{
Sulgi Kim ${ }^{a}$ Mohamad Saad $^{a}$ Debby W. Tsuang ${ }^{b, e}$ Ellen M. Wijsman ${ }^{\text {a, c, d }}$ \\ ${ }^{a}$ Division of Medical Genetics, Department of Medicine, ${ }^{b}$ Department of Psychiatry and Behavioral Sciences, \\ 'Department of Biostatistics, School of Public Health, and d Department of Genome Sciences, School of Medicine, \\ University of Washington, and ${ }^{\mathrm{e}}$ Geriatric Research Education and Clinical Center, Veterans Affairs Puget Sound \\ Health Care System, Seattle, Wash., USA
}

\section{Key Words}

IBD · Linkage analysis · Gene mapping · Sampling subjects . Sequencing $\cdot$ Sequence

\begin{abstract}
Objectives: A particular approach to the visualization of descent of founder DNA copies in a pedigree has been suggested, which helps to understand haplotype sharing patterns among subjects of interest. However, the approach does not provide the information in an ideal format to show haplotype sharing patterns. Therefore, we aimed to find an efficient way to visualize such sharing patterns and to demonstrate that our tool provides useful information for finding an informative subset of subjects for a sequence study. Methods: The visualization package, SharedHap, computes and visualizes a novel metric, the SharedHap proportion, which quantifies haplotype sharing among a set of subjects of interest. We applied SharedHap to simulated and real pedigree datasets to illustrate the approach. Results: SharedHap successfully represents haplotype sharing patterns that contribute to linkage signals in both simulated and real datasets. Using the visualizations we were also able to find ideal sets of subjects for sequencing studies. Conclusions: Our novel metric that can be computed using the SharedHap package provides useful information about haplotype sharing pat-
\end{abstract}

terns among subjects of interest. The visualization of the SharedHap proportion provides useful information in pedigree studies, allowing for a better selection of candidate subjects for use in further sequencing studies.

(c) 2014 S. Karger AG, Basel

\section{Introduction}

Linkage analysis examines sharing patterns of founder genomes among relatives conditional on pedigree information to find a region containing a susceptible variant [1-3]. Mendelian diseases, for which the sharing patterns are the most obvious, have long been successfully studied using linkage analysis [4]. The usual approach to linkage analysis for Mendelian diseases is to investigate a plot of linkage statistics such as LOD scores [1] or - $\log p$ values, but this is only a prelude to the subsequent and more difficult task of identifying susceptible variant(s). Linkage analysis has also been applied to complex traits, where multiple genetic and environmental factors may have an effect on the traits of interest. The presence of allelic and locus heterogeneities makes the study of complex traits more difficult. One approach to tease apart the genetics of complex traits is to study large pedigrees, which may be enriched for rare variants [5-7]. In a large pedigree

\section{KARGER}

E-Mail karger@karger.com

www.karger.com/hhe (c) 2014 S. Karger AG, Basel

0001-5652/14/0781-0001\$39.50/0
Dr. Ellen M. Wijsman

Division of Medical Genetics, Department of Medicine

University of Washington

Box 359460, Seattle, WA 98195-9460 (USA)

E-Mail wijsman@u.washington.edu 
with strong statistical support for linkage, there still is the expectation that there will be shared variants among multiple individuals with similar phenotypes, even in the presence of phenocopies or other heterogeneity.

Recently, high throughput next-generation sequencing technologies that can comprehensively detect basepair level variations have been utilized to identify rare variants in pedigree samples [8]. A cost-effective method with this technology is to perform linkage analysis first and then, based on linkage analysis results, choose individual samples and regions for sequencing [8-13]. The strategy has been used successfully in identifying causative variants in families with Charcot-Marie-Tooth disease [14], Tourette syndrome [15], and exudative vitreoretinopathy [16]. Since next-generation sequencing remains expensive, supplementary genetic information that may increase the chances of identifying causative mutations in complex diseases should be incorporated prior to sequencing.

Inheritance vectors (IVs), which represent the transmission of each founder's diploid DNA copies through the pedigree, can provide more detailed information about the evidence for linkage $[17,18]$. The IVs determine identity-by-descent (IBD) sharing patterns among relatives and along the chromosome, which the linkage score quantifies. Changes in IVs along a chromosome establish the boundaries in a region of interest. However, IVs are seldom obtained deterministically from marker data and are only calculated probabilistically. Probabilistic IV estimates for pedigrees with a wide range of sizes and structures can be obtained by the program gl_auto from the MORGAN package $[19,20]$. This program samples IVs that are consistent with observed genotype data. However, less than fully informative markers and missing genotypes, such as in unsampled subjects in the oldest generations, can contribute to the uncertainty of these estimates. Therefore, the summarization and visualization of these complex IV realizations are necessary to most effectively communicate sharing patterns amongst key individuals in a pedigree.

Marchani and Wijsman [21] discussed the summarization and visualization of IV realizations in a previous publication. They noted the fact that a group of IVs may result in the same IBD sharing pattern even though they differ in the shared set of founder genome [20,22]. Initially, they grouped IV realizations by equivalence classes and then visualized them graphically. A toolset of two programs, IBDgraph and plotIBD, implements this twostage algorithm. Marchani and Wijsman [21] also described how the summarized inheritance patterns can be used to select samples for further sequencing in a study of a complex genetic disease (e.g. late-onset Alzheimer's disease).

The current equivalence-class summarization and visualization scheme, however, does not represent information in an ideal format that demonstrates sharing patterns among subjects of interest. Reliable evidence for linkage in a pedigree should derive from a sharing pattern that is consistent with an excess number of affected relatives sharing an allele IBD. However, the equivalenceclass information can be so detailed that it can obscure the simpler information about the sharing pattern. Also, equivalence classes cannot be simultaneously visualized across multiple markers and across all realizations.

In this paper, we describe an approach to display the visualization of sharing patterns among subjects and along a chromosome. We suggest a metric to represent excess sharing of DNA segments among subjects of interest that can be used as a summary measure for depicting IBD sharing patterns. Computation and visualization is implemented using a two-stage program, ShareHap. We also show that the visualization of this metric is useful specifically for finding a subset of subjects for sequence analysis. The utility of this approach and visualization is demonstrated using simulated and real data examples.

\section{Methods and Results}

\section{Representation of Sharing Patterns}

An IV can be represented by subjects' founder genome labels (FGLs), which are unique identifiers for each of the two haploid genomes in every founder [20,23]. At a particular chromosomal position, the two FGLs of each subject, one inherited from the father and the other from the mother, provide information to determine IBD sharing patterns with other subjects. Positive linkage analysis results indicate excess sharing of FGLs among subjects who also share similar traits. Throughout this paper we assume that the trait is binary, but a continuous trait can be dichotomized with a threshold cutoff so that it can be considered as a binary trait. Among the possible sharing patterns underlying a positive linkage signal, the most obvious pattern would be the situation when all or almost all affected subjects inherited the same FGL. For a given pedigree, the number of affected subjects who inherit the same FGL is typically related to the strength of the linkage evidence at that chromosomal position. The subsequent section describes an algorithm that quantifies the sharing pattern among subjects of interest. 
Fig. 1. Part of the simulated PED52 pedigree from Wijsman et al. [25]. The full PED52 pedigree has been used to infer IVs. However, a partial pedigree that shows the affected subjects (filled symbols) and their relationships is shown here for efficient presentation.

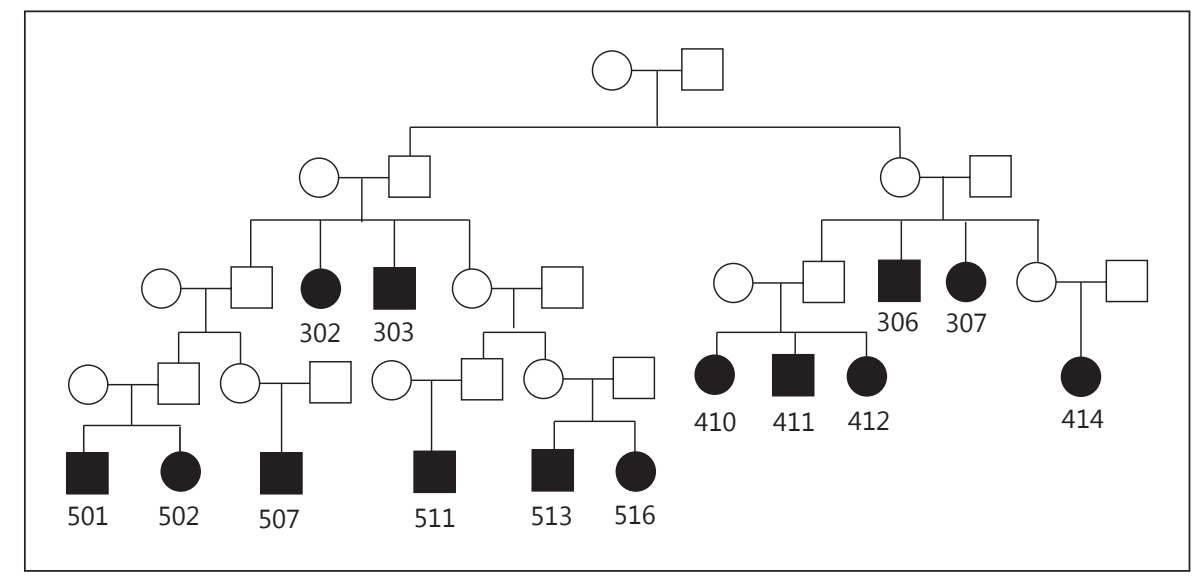

\section{SharedHap: Visualization of Haplotype Sharing}

Pattern

We have written $\mathrm{C}$ and $\mathrm{R}$ programs as two parts of our SharedHap package, which provide the visualization of the sharing patterns from IV realizations with gl_auto in the MORGAN package. SharedHap draws haplotype sharing patterns among subjects selected as a test group. Since we are interested in excess IBD sharing among a group of affected subjects, we began by selecting all affected subjects as a test group. Once we have used the genotypes of all affected and unaffected subjects for inference of IVs and linkage analysis, we need to consider the IV information only from affected subjects. Thus, unless otherwise noted, a subject here denotes an affected subject who is selected as part of a test group.

The program works as follows: for each IV realization, the program identifies the FGL of a marker that is transmitted to the maximum number of subjects in the test group. If multiple FGLs tie, then the program randomly selects one of them. We call this FGL a 'popular FGL' for that specific IV. Once a popular FGL has been identified, we examine each subject to record whether they inherited the popular FGL. The program repeats this process through all IV realizations. The number of times that the subject inherited a popular FGL across all IV realizations is counted and presented as the proportion of the total number of IV realizations. We designate this proportion as the SharedHap proportion, which represents the sharing patterns of a subject with other subjects. The program repeats this computation for multiple markers and then draws a SharedHap graph that depicts the SharedHap proportions of selected subjects and regions.

Setting up a hypothetical haplotype helps interpreting the SharedHap proportion. We imagine a hypothetical

Visualization of Haplotype Sharing

Patterns segment that contains each marker which represents the 'popular' genomes in all IV realizations. Then, we denote a ligation of these segments by the overall term 'popular haplotype'. The SharedHap proportion that we obtained for a subject at a given marker position is the probability that the subject inherited a segment of the popular haplotype at that position.

Parallel plots of the linkage analysis LOD scores and SharedHap proportions for the same region provide information about the relationship between the LOD score curve and haplotype sharing among subjects in the test group. A program in MORGAN, gl_lods, computes the LOD score by a parametric multipoint linkage analysis method [24] from the gl_auto IV realizations that SharedHap uses. SharedHap has an option to read gl_lods output and plot LOD scores above a SharedHap graph.

\section{Simulated Dataset: Haplotype Sharing Pattern}

We illustrated the use of SharedHap on a simulated dataset from Wijsman et al. [25]. A pedigree with a moderate size, PED52, was used as a test case (fig. 1). Genotypes of 67 dense SNP markers at 0.5-cM spacing, spanning $33.5 \mathrm{cM}$, were simulated for 52 subjects. Dichotomized traits were generated using a trait locus located between markers 39 and 40 . The disease allele frequency was set at $20 \%$ and penetrance was set at $(0.05,0.70,0.80)$ for genotypes (dd, Dd, DD), respectively, where D is the disease allele. Note that this model simulates a complex disease, which is not fully explained by this disease allele, and therefore incorporates the possibility of phenocopies into our illustration of the SharedHap approach. Further details about the simulation are described in Wijsman et al. [25]. Among 100 replicates, we chose a dataset that had one of the largest parametric multipoint LOD scores of 
Fig. 2. LOD scores from a parametric linkage analysis (a) and the corresponding SharedHap graph (b) for the analysis of PED52.

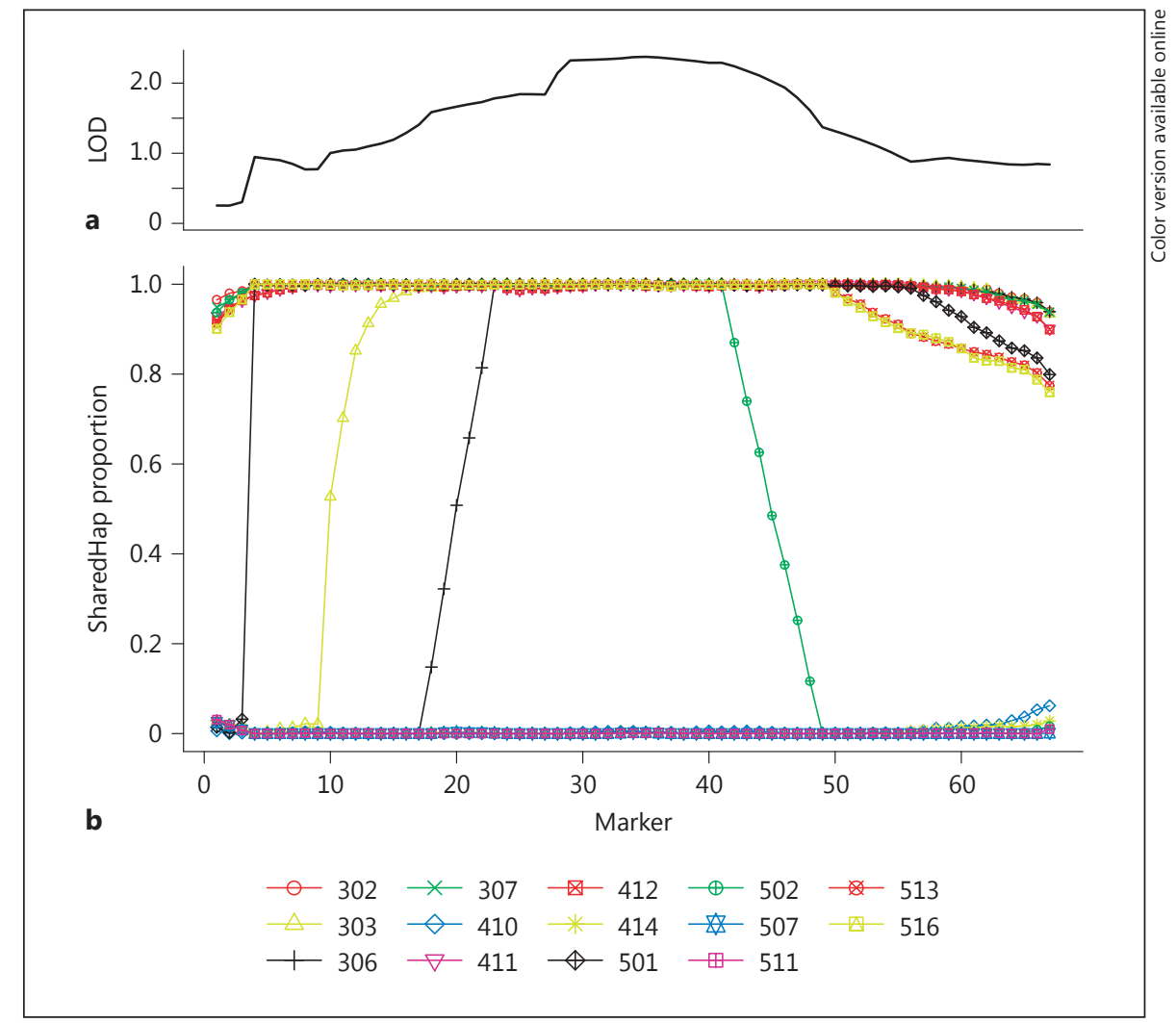

about 2.4, based on the true underlying trait model parameters (fig. 2a). After 3,000 burn-in scans, we used 30,000 MCMC IV realizations to sample 1,000 IVs by scoring every 30 th IV realization. All 14 affected subjects of the full set of 52 subjects were used as the test group.

The parallel plots in figure 2 deliver the following information: the LOD score curve in figure $2 \mathrm{a}$ was computed with the same parameters that were used for generating the data. There is evidence for linkage across markers 18-50, defined as the region of interest with $\mathrm{LOD}>\left(\mathrm{LOD}_{\mathrm{MAX}}-1\right)$ [26], where $\mathrm{LOD}_{\mathrm{MAX}}$ is the maximum observed LOD score in the region under consideration. Figure $2 \mathrm{~b}$ shows the SharedHap proportions. A subject inherits a segment of the popular haplotype in a region where the subject has a SharedHap proportion near 1. For example, subject 303 inherited the popular haplotype in a region from about marker 12 to the right end where the subject has a SharedHap proportion $>0.8$. Therefore, two subjects would share a segment of popular haplotype for the region where both have high SharedHap proportions. For example, subjects 303 and 502 share the popular haplotype for the region spanning approximately markers $12-42$.
The most obvious sharing pattern is in a region spanning markers 23-42 where the greatest number of subjects share the popular haplotype. We denote this region as the 'maximum sharing region'. The region loosely overlaps the region with the linkage signal defined earlier. This implies that the maximum sharing by affected subjects is likely to have driven the linkage signal that is computed for all affected and unaffected subjects. Note that the maximum sharing region includes the trait locus, which is located between markers 39 and 40 .

\section{Simulated Dataset: Sampling Subjects for Sequencing}

The haplotype shared by the greatest number of affected subjects is expected to harbor a susceptibility variant for a trait in or near the region with maximum sharing across affected subjects. To identify the variant, a subset of subjects may be selected and can be sequenced for the whole genome or a targeted region. Characteristics of variants on this shared haplotype, such as location or allele frequency in the population, might be considered in later analyses.

The selection of candidate subjects for sequencing can have a major impact on the outcome of a sequencing 
study [12]. The affected factors include the probability of finding the target variant, statistical significance for the discovered variants, and size of the search area for the variant. Therefore, the selection of the ideal subjects is critical.

We can select an ideal subset based on sharing patterns visualized by the SharedHap package. In this paper, we consider only sharing patterns when sampling subjects for sequencing even though there are other factors that could also be considered, such as cost or DNA quality or quantity. A similar sampling method has previously been suggested, but is limited to collecting single subjects selected from nuclear families [27] - as opposed to the more general pedigrees handled by SharedHap. In this section, for simplicity, we consider selecting a subset of subjects for target-region sequencing, where we consider a single region. The ideal subset of subjects should have the following characteristics in a linkage region under consideration: (1) the subset of subjects has a shared segment in a maximum sharing region that appears to contribute to a linkage region, and (2) the shared segment is relatively short, resulting in a small, but reliable, search area in a follow-up sequence study. A smaller search area contributes to the efficiency of a study because it requires the investigation of fewer candidate variants. The second condition will typically also prevent the selection of close relatives who are likely to share a long segment by chance alone [28].

As an example, we use a subset of size two to demonstrate an efficient way to select an ideal subset that satisfies both conditions above. We can identify two subjects whose SharedHap proportions sharply decrease substantially at each end of the maximum sharing region. This subset would generally satisfy the two conditions. Because these two subjects share a popular haplotype in the maximum sharing region, this subset would satisfy the first condition. It is straightforward for the second condition, because we chose two subjects as satisfying the condition.

Assume that we wish to find an ideal subset of size two subjects in our simulated dataset. We identified earlier that the maximum sharing region, which is the region spanning markers $23-43$, appears to have driven the linkage region spanning markers $18-50$ (fig. 2). Figure $2 \mathrm{~b}$ shows that subjects 306 and 502 delimit the maximum sharing region at each end. We might, therefore, choose the set of subjects 306 and 502 as an ideal subset for sequencing. In fact, investigating the masked information revealed that these selected subjects inherited the same FGL at the trait locus. The two subjects are distant rela-

Visualization of Haplotype Sharing

Patterns

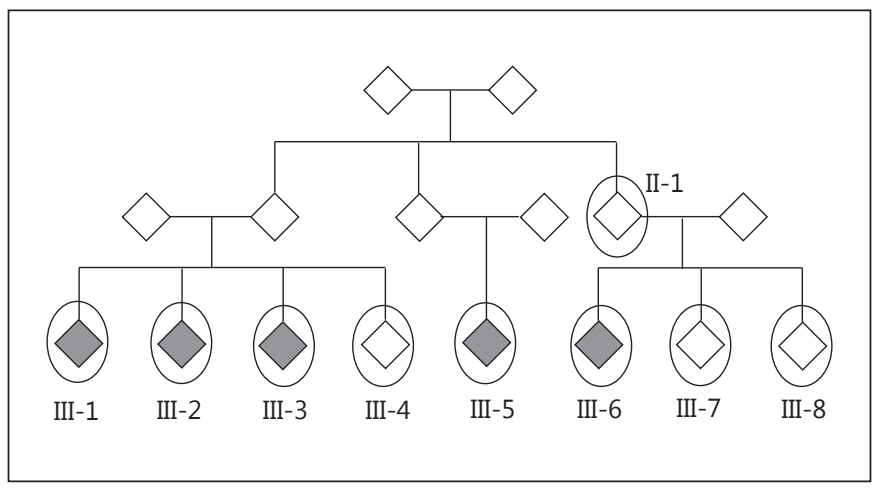

Fig. 3. Pedigree of our real data. Filled diamonds denote the affected individuals, and empty diamonds denote the unaffected individuals. We genotyped the circled individuals. Genders are intentionally hidden.

tives - sixth-degree relatives (fig. 1) - whose sharing in the maximum sharing region is unlikely to have occurred by chance.

Sampling for multiple-region or genome-wide sequencing requires considering multiple linkage regions. A similar approach can be used to find an ideal subset that best satisfies the two conditions (1) and (2) for each linkage region. This will be illustrated with a real data example in the next section.

\section{Real Dataset: Haplotype Sharing Pattern and}

Sampling Subjects for Sequencing

We applied SharedHap to a real study of schizophrenia. An aim of the study was to identify a few subjects for further genome-wide sequencing to find a susceptibility variant in a region with evidence of linkage. Pedigree samples from previous studies $[29,30]$ were genotyped with 4,604 genome-wide SNP markers spaced at 1-cM intervals, on average, selected from the Illumina HumanLinkage-24 BeadChip array. Our nonparametric and parametric multipoint linkage analyses $[1,3,31]$ identified a pedigree of interest that has evidences for linkage on chromosomes 14 and 19 genotyped for 185 and 100 markers, respectively. The pedigree structure is shown in figure 3 . We initially considered chromosome 14 for sampling an ideal subset.

Figure 4 shows the results of the parametric linkage analysis and the SharedHap graph for the pedigree on chromosome 14. Parameters for the linkage analysis were set as follows: a frequency of the disease allele $\mathrm{d}$ of $1 \%$, and penetrance of $(0.005,0.300,0.600)$ for genotypes (dd, Dd, DD). For the SharedHap graph, we chose a test group 
Fig. 4. Parallel plots of the results of the linkage analysis (a) and the corresponding SharedHap graph (b) on chromosome 14 for the real data.

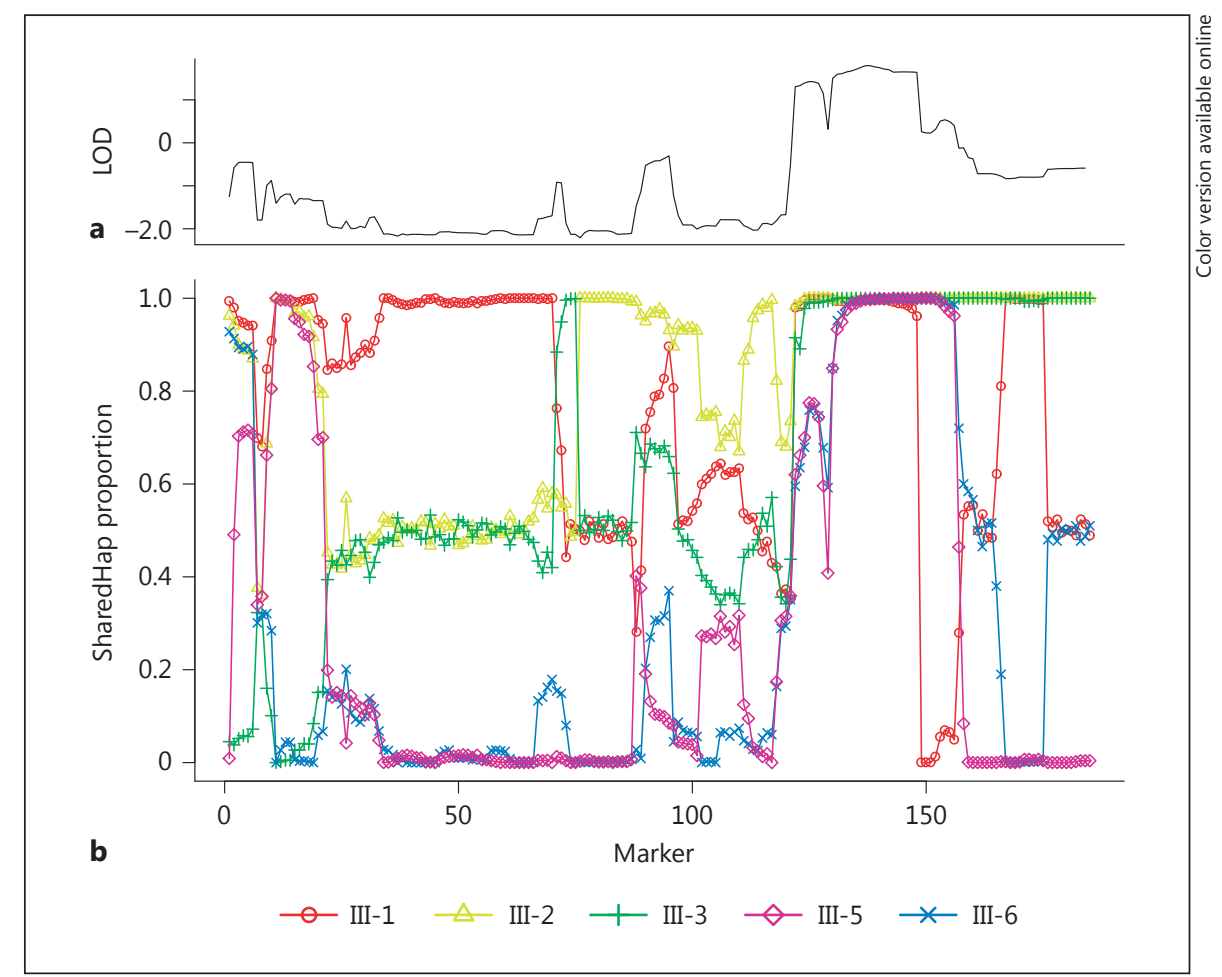

of all five affected subjects. The linkage region spans markers $125-150$ and the maximum sharing region spans markers 130-150. The overlap of the two regions indicates that the linkage signal was driven by haplotype sharing of these five affected subjects. The haplotype sharing of subject III-1 delimits the right end of the maximum sharing region. At the same time, either subject III-5 or III-6 delimits the left end of the maximum sharing region. Therefore, any pair of III-1 and either of III-5 or III-6 would be an ideal subset for sequencing. On chromosome 19 , subject III-6 alone delimits the maximum sharing region, which spans markers 72-82 (fig. 5). In consideration of the two linkage regions, we might choose III-1 and III- 6 as our candidate set for sequencing. Note that our sampling strategy avoids sampling of parent-offspring or sibling pairs (fig. 3), both of whom are likely to share a segment even when there is no relationship with the disease.

\section{Discussion}

We introduced the use of a measure, the SharedHap proportion, for summarizing and visualizing IBD sharing patterns. The SharedHap graph, which is a visualization of SharedHap proportions in a test group, describes IBD sharing patterns within this test group, as we illustrated with simulated and real pedigree data. We also demonstrated that parallel graphs of a linkage result and a SharedHap graph can be used for sampling an ideal subset for target-region and genome-wide sequencing. In the context of a trait defined by dichotomization of a quantitative trait, sharing patterns based on different sample sizes and dichotomization thresholds can be investigated. For example, additional subjects with a less extreme quantitative trait could be included. We would expect that affected subjects would be less likely to share a common haplotype in this case compared to selecting subjects with more extreme trait values.

Informative markers give benefits to our approach as they also do with other linkage studies. A region with less informative markers would likely result in some or all subjects having uncertain SharedHap proportions, i.e. intermediate values between 0 and 1 . In our examples this would be intermediate values for III-2 and III-3 in the region around $50 \mathrm{cM}$ in figure 3.

We have shown that the SharedHap graph successfully enables the visualization of shared genomic regions and flanking recombinant events where there is linkage evidence for dominantly or additively inherited variants. 
Fig. 5. Parallel plots of the results of the linkage analysis (a) and the corresponding SharedHap graph (b) on chromosome 19 for the real data.

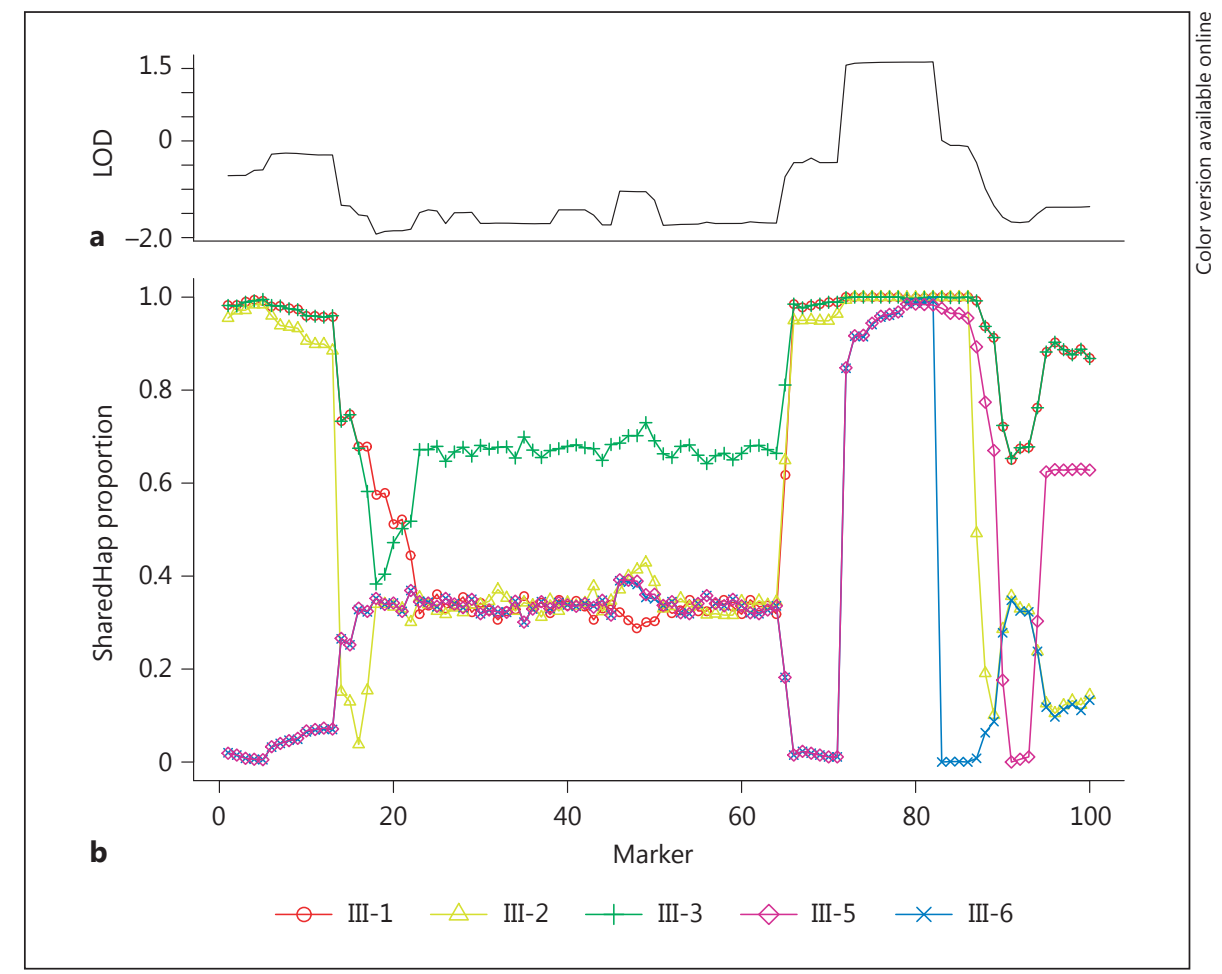

However, for recessive models, despite clear sharing near the trait locus, the boundaries of regions of interest defined by the SharedHap graph may not present clear deviations from high or low sharing. This is because there will be two competing equally popular haplotypes. Each will be shared by most affected subjects near the trait locus, but which will have different flanking recombinant events. Genetic heterogeneity within pedigrees would also reduce the sharing, resulting in less obvious sharing patterns, although when used in conjunction with a strong linkage signal, it is unlikely that this will be a major complication.

SharedHap is implemented as a pair of $\mathrm{C}$ and $\mathrm{R}$ programs. The $\mathrm{C}$ program computes the SharedHap proportions and the $\mathrm{R}$ program draws a plot of these proportions. The program and our simulated dataset can be found at: http://faculty.washington.edu/wijsman/ software.shtml.

SharedHap inherits the utility of previous methods implemented in IBDgraph and plotIBD, with advantages over these earlier programs. The previous approach shows the IV realizations as equivalence classes, which may not be the optimal information for use in simplifying the information needed to select a set of subjects of interest. In contrast, SharedHap further simplifies the inheri- tance pattern, focusing on the sharing of a popular haplotype among a test group of subjects. This is of direct interest for selecting subjects. The information can be shown for multiple IVs and for multiple markers in one plot, which was not possible with the previous methods. In our example, a plot was able to represent a sharing pattern from 1,000 IVs typed with 5,000 markers. The SharedHap package, therefore, can save time and effort in identifying the markers that show the most promising sharing patterns among subjects of interest.

The SharedHap graph can also be used for other questions than those illustrated here. For example, it can be used for determining the choice of a sequencing technology between targeted sequencing and whole-genome/ whole-exome sequencing. The presence of a single region that shows a highly promising sharing pattern would suggest further use of targeted-sequencing technology, while the lack of such a region might suggest whole-genome/ whole-exome sequencing because there seems to be extensive disease heterogeneity. In conclusion, the visualization of haplotype sharing patterns through the SharedHap graph provides detailed information underlying linkage signals that can be utilized for many purposes in pedigree studies. 


\section{Acknowledgements}

This work was supported by a US Department of Veterans Affairs, Office of Research Development, Biomedical Laboratory Re- search merit review; VISN-20 MIRECC, and NIH grants No. R01MH065558 and P50AG005136. The funding agencies did not play a role in the study design, data collection and analysis, decision to publish, or preparation of the manuscript.

\section{References}

1 Morton NE: Sequential tests for the detection of linkage. Am J Hum Genet 1955;7:277-318

2 Ott J: Analysis of Human Genetic Linkage, ed 3. Baltimore, Johns Hopkins University Press, 1999.

3 Kruglyak L, Daly MJ, Reeve-Daly MP, Lander ES: Parametric and nonparametric linkage analysis: a unified multipoint approach. Am J Hum Genet 1996;58:1347-1363.

4 Botstein D, Risch N: Discovering genotypes underlying human phenotypes: past successes for Mendelian disease, future approaches for complex disease. Nat Genet 2003;33:228-237.

5 Wijsman EM, Amos CI: Genetic analysis of simulated oligogenic traits in nuclear and extended pedigrees: summary of GAW10 contributions. Genet Epidemiol 1997;14:719735.

6 Manolio TA, Collins FS, Cox NJ, et al: Finding the missing heritability of complex diseases. Nature 2009;461:747-753.

7 Wijsman EM: The role of large pedigrees in an era of high-throughput sequencing. Hum Genet 2012;131:1555-1563.

-8 Bailey-Wilson JE, Wilson AF: Linkage analysis in the next-generation sequencing era. Hum Hered 2011;72:228-236.

9 Allen-Brady K, Farnham J, Cannon-Albright L: Strategies for selection of subjects for sequencing after detection of a linkage peak. BMC Proc 2011;5(suppl):S77.

10 Bowden DW, An SS, Palmer ND, Brown WM, Norris JM, Haffner SM, Hawkins GA, Guo X, Rotter JI, Chen YDI, Wagenknecht LE, Langefeld CD: Molecular basis of a linkage peak: exome sequencing and family-based analysis identify a rare genetic variant in the ADIPOQ gene in the IRAS family study. Hum Mol Genet 2010;19:4112-4120.

11 Cirulli ET, Goldstein DB: Uncovering the roles of rare variants in common disease through whole-genome sequencing. Nat Rev Genet 2010;11:415-425.

12 Shi G, Rao DC: Optimum designs for nextgeneration sequencing to discover rare variants for common complex disease. Genet Epidemiol 2011;35:572-579.

13 Choi SH, Liu C, Dupuis J, Logue MW, Jun G: Using linkage analysis of large pedigrees to guide association analyses. BMC Proc 2011; 5(suppl):S79.
4 Weedon MN, Hastings R, Caswell R, Xie WJ, Paszkiewicz K, Antoniadi T, Williams M, King C, Greenhalgh L, Newbury-Ecob $\mathrm{R}$, Ellard S: Exome sequencing identifies a DYNC1H1 mutation in a large pedigree with dominant axonal Charcot-Marie-Tooth disease. Am J Hum Genet 2011;89:308-312.

15 Sundaram SK, Huq AM, Sun Z, Yu W, Bennett L, Wilson BJ, Behen ME, Chugani HT: Exome sequencing of a pedigree with Tourette syndrome or chronic tic disorder. Ann Neurol 2011;69:901-904.

16 Nikopoulos K, Gilissen C, Hoischen A, van Nouhuys CE, Boonstra FN, Blokland EAW, Arts P, Wieskamp N, Strom TM, Ayuso C, Tilanus MAD, Bouwhuis S, Mukhopadhyay A, Scheffer H, Hoefsloot LH, Veltman JA, Cremers FPM, Collin RWJ: Next-generation sequencing of a $40 \mathrm{Mb}$ linkage interval reveals TSPAN12 mutations in patients with familial exudative vitreoretinopathy. Am J Hum Genet 2010;86:240-247.

17 Kruglyak L, Lander ES: Complete multipoint sib-pair analysis of qualitative and quantitative traits. Am J Hum Genet 1995;57:439-454.

18 Lander ES, Green PJ: Construction of multilocus genetic maps in humans. Proc Natl Acad Sci USA 1987;84:2363-2367.

19 MORGAN: a package for Markov chain Monte Carlo in genetic analysis (version 3.1.1), 2012, http://www.stat.washington.edu/thompson/Genepi/MORGAN/Morgan.shtml.

20 Thompson EA: The structure of genetic linkage data: from LIPED to $1 \mathrm{M}$ SNPs. Hum Hered 2011;71:86-96.

21 Marchani EE, Wijsman EM: Estimation and visualization of identity-by-descent within pedigrees simplifies interpretation of complex trait analysis. Hum Hered 2011;72:289297.

22 Koepke H, Thompson E: Efficient identification of equivalences in dynamic graphs and pedigree structures. J Comput Biol 2013;20: 551-570.
23 Thompson EA: MCMC in the analysis of genetic data on pedigrees; in Kendall WS, Wang JS, Liang F (eds): Markov Chain Monte Carlo: Innovations and Applications. Singapore, World Scientific Publishing Company, 2005.

24 Tong LP, Thompson E: Multilocus lod scores in large pedigrees: combination of exact and approximate calculations. Hum Hered 2008; 65:142-153.

25 Wijsman EM, Rothstein JH, Thompson EA: Multipoint linkage analysis with many multiallelic or dense diallelic markers: MCMC provides practical approaches for genome scans on general pedigrees. Am J Hum Genet 2006; 79:846-858.

26 Conneally PM, Edwards JH, Kidd KK, Lalouel JM, Morton NE, Ott J, White R: Report of the committee on methods of linkage analysis and reporting. Cytogenet Cell Genet 1985;40: 356-359.

27 Fingerlin TE, Boehnke M, Abecasis GR: Increasing the power and efficiency of diseasemarker case-control association studies through use of allele-sharing information. Am J Hum Genet 2004;74:432-443.

28 Akula N, Detera-Wadleigh S, Shugart YY, Nalls M, Steele J, McMahon FJ: Identity-bydescent filtering as a tool for the identification of disease alleles in exome sequence data from distantrelatives. BMCProc2011;5(suppl):S76.

29 Cloninger CR, Kaufmann CA, Faraone SV, Malaspina D, Svrakic DM, Harkavy-Friedman J, Suarez BK, Matise TC, Shore D, Lee H, Hampe CL, Wynne D, Drain C, Markel PD, Zambuto CT, Schmitt K, Tsuang MT: Genome-wide search for schizophrenia susceptibility loci: the NIMH Genetics Initiative and Millennium Consortium. Am J Med Genet 1998;81:275-281.

30 Tsuang MT, Faraone SV, Bingham S, Young K, Prabhudesai S, Haverstock SL, Mena F, Menon AS, Pepple J, Johnson J, Baldwin C, Weiss D, Collins J: Department of Veterans Affairs Cooperative Studies Program genetic linkage study of schizophrenia: ascertainment methods and sample description. Am J Med Genet 2000;96:342-347.

- 31 Abecasis GR, Cherny SS, Cookson WO, Cardon LR: Merlin - rapid analysis of dense genetic maps using sparse gene flow trees. Nat Genet 2002;30:97-101. 